\title{
Olmesartan is not associated with the risk of enteropathy: a Korean nationwide observational cohort study
}

\author{
Seng Chan You ${ }^{1,}$, Hojun Park ${ }^{2,}$, Dukyong Yoon ${ }^{1}$, Sooyoung Park ${ }^{2}$, Boyoung Joung ${ }^{3}$, and Rae Woong Park ${ }^{1,4}$
}

${ }^{1}$ Department of Biomedical Informatics, Ajou University School of Medicine, Suwon; ${ }^{2}$ Department of Statistics, Ewha Womans University, Seoul; ${ }^{3}$ Division of Cardiology, Department of Internal Medicine, Yonsei University College of Medicine, Seoul; ${ }^{4}$ Department of Biomedical Sciences, Ajou University Graduate School of Medicine, Suwon, Korea

Received: December 27, 2016

Revised : June 12, 2017

Accepted: June 12, 2017

\section{Correspondence to}

Rae Woong Park, M.D.

Department of Biomedical Informatics, Ajou University School of Medicine, 206 World cup-ro, Yeongtong-gu,

Suwon 16499, Korea

Tel: $+82-31-219-4471$

Fax: +82-31-219-4472

E-mail:veritas@ajou.ac.kr

*These authors contributed equally to this work.
Background/Aims: Olmesartan, a widely used angiotensin II receptor blocker (ARB), has been linked to sprue-like enteropathy. No cases of olmesartan-associated enteropathy have been reported in Northeast Asia. We investigated the associations between olmesartan and other ARBs and the incidence of enteropathy in Korea.

Methods: Our retrospective cohort study used data from the Korean National Health Insurance Service to identify 108,559 patients (58,186 females) who were initiated on angiotensin converting enzyme inhibitors (ACEis), olmesartan, or other ARBs between January 2005 and December 2012. The incidences of enteropathy were compared among drug groups. Changes in body weight were compared after propensity score matching of patients in the ACEis and olmesartan groups. Results: Among 108,559 patients, 31 patients were diagnosed with enteropathy. The incidences were $0.73,0.24$, and 0.37 per 1,000 persons, in the ACEis, olmesartan, and other ARBs groups, respectively. Adjusted rate ratios for enteropathy were: olmesartan, 0.33 (95\% confidential interval [CI], 0.10 to $1.09 ; p=0.070$ ) and other ARBs, 0.34 ( $95 \% \mathrm{CI}, 0.14$ to $0.83 ; p=0.017$ ) compared to the ACEis group after adjustment for age, sex, income level, and various comorbidities. The post hoc analysis with matched cohorts revealed that the proportion of patients with significant weight loss did not differ between the ACEis and olmesartan groups.

Conclusions: Olmesartan was not associated with intestinal malabsorption or significant body weight loss in the general Korean population. Additional largescale prospective studies of the relationship between olmesartan and the incidence of enteropathy in the Asian population are needed.

Keywords: Angiotensin receptor antagonists; Olmesartan; Insurance claim review; Drug-related side effects and adverse reactions; Intestinal diseases

\section{INTRODUCTION}

Olmesartan medoxomil, the angiotensin II receptor blocker (ARB), has been widely used to manage cardiovascular diseases including hypertension since its approval in 2002 [1] with estimated worldwide sales of US
\$2 billion in 2009. Rubio-Tapia et al. [2] first described a case series of severe sprue-like enteropathy at the Mayo Clinic in the United States in 2012, which was linked to the use of olmesartan. Similar cases have been reported at other locations in the United States, Europe [3], and India [4]. A class effect of ARBs was suspected after sever- 
al case reports of other ARB-associated enteropathy $[5,6]$.

In July 2013, the United States Federal Drug Administration changed the olmesartan medoxomil combination label to include warnings of sprue-like enteropathy [7]. Recently, a French nationwide cohort study found an association between olmesartan and the risk of enteropathy, using claim data [8]. In April 2016, Agence Nationale de Sécurité du Médicament et des Produits de Santé(The national agency for the safety of medicines and health products) delisted specialties containing olmesartan in France because of the risk of sprue-like enteropathy and its unproven effectiveness in terms of improving morbidity and mortality related to hypertension.

The prevalence of celiac disease is very low or unknown in Northeast Asian countries such as Korea, Japan, and China [9]. To date, no cases of olmesartan- or other ARB-associated sprue-like enteropathy have been reported in Northeast Asia. We investigated the associations of olmesartan and other ARBs with enteropathy in Korea. We investigated the associations of olmesartan and other ARBs with enteropathy in Korea using a large nationwide cohort. We also compared changes in weight, hemoglobin, and serum cholesterol levels in patients taking angiotensin converting enzyme inhibitors (ACEis) and olmesartan.

\section{METHODS}

\section{Data source}

The Korean National Health Insurance Service (NHIS) was established in 1977 and provided universal coverage by 1989 [10]. In 2015, the NHIS released a fully anonymized, de-identified National Sample Cohort 2002 to 2013 database (NHIS-NSC 2002-2013). A systematic stratified random sampling strategy with proportional allocation in each stratum was used to provide representative, useful health insurance data. The cohort consisted of 1,025,320 Koreans identified in 2002 and followed for 11 years through 2013. The database contains demographic information, diagnoses, prescriptions from clinics and/or hospitals, ambulatory healthcare use, hospitalization, and health outcomes such as death for the respective years [11].

The NHIS instituted the biennial national health examination program, which is recommended for all of the insured employees or self-employed individuals over 40 years old and their dependents. The national health examination data include body weight, height, hemoglobin, and total cholesterol measurements obtained on the same day after an overnight fast. These data are included in the NHIS-NSC 2002 to 2013. We could not obtain informed consent from individual participants because the dataset was fully anonymized and de-identified. Detailed information on the NHIS-NSC database has been previously published [11]. Our study was performed in accordance with the ethical guidelines of the 1975 Declaration of Helsinki, and was approved by the Institutional Review Board of Ajou University Hospital (AJIRB-MED-EXP17). Because of retrospective nature of this study, the informed consent was not required.

\section{Study population and exposure assessment}

Olmesartan was introduced in Korea in 2005. We enrolled NHIS-NSC 2002 to 2013 patients who were prescribed ACEis, olmesartan, or other ARBs between January 2005 and December 2012. All of the patients who started or were using ACEis or ARBs between 2002 and 2004 were excluded from the study to prevent left censoring. Only patients who had observation period more than 1 year before initiation of these drugs were included. Furthermore, patients were excluded from the analysis if they had been diagnosed with intestinal malabsorption prior to the initiation of ACEis or ARBs. Because celiac disease is rare in Korea, no established treatment regime exists; thus, we could not exclude patients based on treatment for this disease. The study sample was divided into three groups according to drug exposure (olmesartan, other ARBs, or ACEis). Each group was mutually exclusive. Because adverse events may necessitate a change in drugs, the olmesartan and other ARBs groups included patients who had previously taken ACEis. The period of overlapping exposures (e.g., ACEi + olmesartan) was removed from the analysis to prevent misinterpretation of the data. Exposure was calculated starting from the date a prescription was filled for a drug of interest more than 7 days. The end of exposure was defined as the end of the prescription with an additional 30-day grace period to account for incomplete medication adherence and to avoid the underestimation of drug exposure or misinterpretation of adverse events. 


\section{Outcome and study covariates}

Disease information was classified according to the Korean Classification of Disease 6 (KCD-6), which is based on the 1oth revision of the International Classification of Diseases 10 (ICD-10) code. The primary outcome was diagnosis and treatment for intestinal malabsorption (KCD-6 code, K90x). The following comorbidities were selected as covariates based on a previous French nationwide study [8]: heart failure, dementia, diabetes mellitus (DM), autoimmune diseases (rheumatoid arthritis, Hashimoto thyroiditis, IgA deficiency, dermatitis herpetiformis, systemic lupus erythematosus, Sjogren's syndrome, dermatopolymyositis, complement deficiency, angioedema, and inflammatory bowel diseases), organ transplantation, cancer, and chronic kidney disease (CKD). In addition, level of average income, hypertension and dyslipidemia were also adjusted. Patients with autoimmune diseases are prone to develop celiac disease or other autoimmune diseases. ACEis and ARBs are frequently prescribed for DM. Diarrhea, caused by diabetic autonomic neuropathy is a common complication of DM. Heart failure, CKD, cancer, and organ transplantation are serious comorbidities that may have an impact on malabsorption and diarrhea.

\section{Post hoc analysis using a matched cohort}

To reduce bias caused by the underdiagnosis of subclinical intestinal malabsorption, we compared the changes in body weight, cholesterol, and hemoglobin, and the incidence of significant body weight loss defined by annually $5 \%$ or more body weight loss [12] in the olmesartan and ACEis groups within those of a matched cohort. The R package MatchIt ( $R$ Foundation for Statistical Computing, Vienna, Austria) was used to generate propensity scores and match olmesartan and ACEis users who underwent general medical examinations 1 year before drug initiation and while under treatment with the drug. Propensity scores, which represent the probability of receiving each drug, were used in the logistic regression analysis [13]. Seventeen covariates were used to generate the propensity score (age, sex, income level, initial examination time, total duration of drug use, differences in the year of the physical examination, baseline systolic blood pressure, fasting blood glucose, total cholesterol, hemoglobin, body mass index [BMI], hypertension, dyslipidemia, heart failure, chronic kidney disease, ongoing cancer, and income level). After the propensity scores were generated, the patients in each drug group underwent 1:1 nearest neighbor (Greedy-type) matching of the logit of the propensity score with a caliper width of a 0.05 standard deviation of the score. Matching was performed without replacement, and non-matched results were discarded.

\section{Statistical analysis}

A multivariable logistic regression model was used to analyze the incidence of enteropathy associated with drug after adjustment for selected comorbidities. The means or percentages of baseline characteristics, and the results from physical examination between drug groups were compared using independent Student $t$ tests for continuous variables and the chi-square for categorical variables. Statistical tests were conducted using $\mathrm{R}$ version 3.2.5 ( $\mathrm{R}$ Foundation). All $p$ values were twotailed, and $p$ values $<0.05$ were considered statistically significant.

\section{RESULTS}

\section{Study sample}

We identified 108,687 patients from the NHIS-NSC (2002 to 2013) database who were initiated on ACEis, olmesartan, or other ARBs between 2005 and 2012. We excluded 128 patients diagnosed with enteropathy prior to inclusion in the database. A total of 108,559 patients were enrolled in our study, culminating in total observation period of 1,263,601 person-years (PYs) including observation period prior to initiation of the drug. The patients were grouped according to drug exposure: ACEis, 8,487 patients (females, 45.4\%); olmesartan, 23,610 patients (females, $47.9 \%$ ); and ARBs, 76,462 patients (females, $48.4 \%$ ) (Fig. 1). Patient baseline characteristics are shown in Table 1.

Patients of ACEis group were older and had more heart failure, dementia, DM, autoimmune disease, organ transplantation, ongoing cancer, and CKD compared to olmesartan group (all $p<0.05$ ). Drug use period was longer in olmesartan group than in ACEis group (1.60 years vs. 1.50 years, $p<0.001$ ). 


\section{Incidence of enteropathy and risk over time}

A total of 31 patients were diagnosed with enteropathy during drug use (Table 2). The incidences of enteropathy in the ACEis, olmesartan, and other ARBs groups were $0.73,0.24$, and 0.37 per 1,000 person, respectively. Among patients exposed to a drug for more than 1 year, the incidences of enteropathy in the ACEis, olmesartan, and other ARBs groups were 0.99, 0.18, and 0.19 per 1,000 persons, respectively. The crude odd ratios were lower in the olmesartan and other ARBs group than in ACEis group (odd ratio [OR], 0.26; 95\% confidential interval [CI], 0.08 to $0.80 ; p=0.020$ in olmesartan group and OR, $0.30 ; 95 \% \mathrm{CI}$, 0.13 to $0.72 ; p=0.007$ in other ARBs group) (Table 3).

Adjusted rate ratios for enteropathy were: olmesartan,
0.33 (95\% CI, 0.10 to 1.09; $p=0.070$ ) and other ARBs, 0.34 (95\% CI, 0.14 to $0.83 ; p=0.017$ ) compared to the ACEis

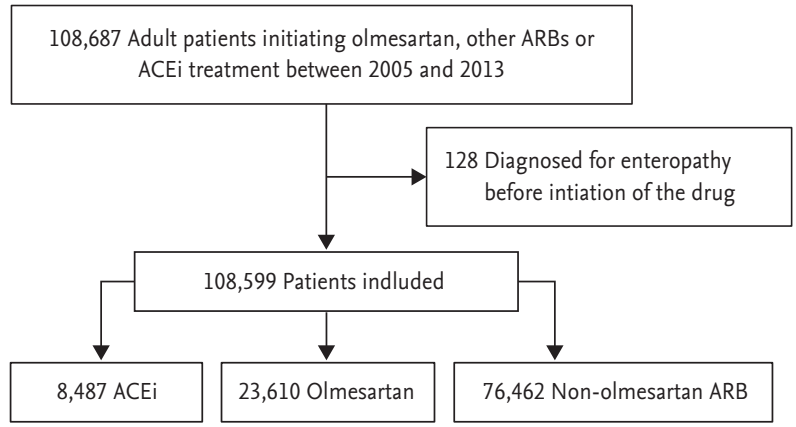

Figure 1. Inclusion flow chart. ACEi, ACE inhibitor; ARB, angiotensin receptor blocker.

Table 1. Baseline characteristics

\begin{tabular}{|c|c|c|c|c|}
\hline Variable & $\operatorname{ACEi}(\mathrm{n}=8,487)$ & Olmesartan $(n=23,610)$ & Other ARBs $(n=76,462)$ & $p$ value $^{a}$ \\
\hline Age, yr & $62.2 \pm 14.3$ & $58.9 \pm 13.3$ & $58.9 \pm 13.3$ & $<0.001$ \\
\hline Female sex & $3,856(45.4)$ & $11,309(47.9)$ & $37,021(48.4)$ & $<0.001$ \\
\hline Income level, deciles & $5.44 \pm 2.73$ & $5.36 \pm 2.67$ & $5.42 \pm 2.70$ & 0.037 \\
\hline Total observation period, yr & $10.8 \pm 2.2$ & $11.7 \pm 1.1$ & $11.7 \pm 1.1$ & $<0.001$ \\
\hline Drug use period, yr & $1.50 \pm 2.16$ & $1.60 \pm 1.82$ & $2.46 \pm 2.1$ & $<0.001$ \\
\hline \multicolumn{5}{|l|}{ Comorbidity } \\
\hline Hypertension & $6,684(78.8)$ & $21,882(92.7)$ & $69,983(91.5)$ & $<0.001$ \\
\hline Dyslipidemia & $2,510(29.6)$ & $7,422(31.4)$ & $26,565(34 \cdot 7)$ & 0.002 \\
\hline Heart failure & $875(10.3)$ & $1,072(4.5)$ & $3,729(4.9)$ & $<0.001$ \\
\hline Dementia & $229(2.7)$ & $526(2.2)$ & $1,291(1.7)$ & 0.016 \\
\hline Diabetes mellitus & $2,450(28.9)$ & $6,176(26.2)$ & $19,643(25 \cdot 7)$ & $<0.001$ \\
\hline Autoimmune disease & $87(1.0)$ & $182(0.8)$ & $787(1.0)$ & 0.033 \\
\hline Organ transplantation & $27(0.3)$ & $11(0.0)$ & $76(0.1)$ & $<0.001$ \\
\hline Ongoing cancer & $622(7 \cdot 3)$ & $611(2.6)$ & $2,393(3.1)$ & $<0.001$ \\
\hline Chronic kidney disease & $76(0.9)$ & $220(0.9)$ & $655(0.9)$ & 0.815 \\
\hline \multicolumn{5}{|l|}{ Inclusion year } \\
\hline 2005 & $1,716(20.2)$ & $1,630(6.9)$ & $5,680(7 \cdot 4)$ & \\
\hline 2006 & $1,413(16.6)$ & $2,752(11.7)$ & $6,180(8.1)$ & \\
\hline 2007 & $1,539(18.1)$ & $3,476(14 \cdot 7)$ & $6,442(8.4)$ & \\
\hline 2008 & $1,425(16.8)$ & $3,738(15.8)$ & $9,387(12.3)$ & \\
\hline 2009 & $870(10.3)$ & $3,020(12.8)$ & $13,861(18.1)$ & \\
\hline 2010 & $534(6.3)$ & $2,963(12.5)$ & $11,237(14.7)$ & \\
\hline 2011 & $522(6.2)$ & $3,049(12.9)$ & $12,738(16.7)$ & \\
\hline 2012 & $468(5.5)$ & $2,982(12.6)$ & $10,937(14 \cdot 3)$ & \\
\hline
\end{tabular}

Values are presented as mean \pm SD or number (\%).

$\mathrm{ACEi}$, angiotensin converting enzyme inhibitor; $\mathrm{ARB}$, angiotensin receptor blocker.

${ }^{\mathrm{a}}$ The results from ACEi group and olmesartan group were compared. 
group after adjustment age, sex, income level, hypertension, dyslipidemia, heart failure, dementia, DM, autoimmune diseases, CKD, organ transplantation, and ongoing cancer. Stratification of the patients according to duration of drug exposure revealed that the adjusted risk of enteropathy in patients receiving olmesartan for more than 1 year was not significantly different from that of patients receiving ACEis (OR, 0.25; $95 \% \mathrm{CI}, 0.04$ to 1.62; $p=0.147)$. Adjusted OR was lower in patients using other ARBs more than 1 year than in patients using ACEi (OR, $0.24 ; 95 \% \mathrm{CI}, 0.06$ to $0.90 ; p=0.034$ ). The risks of enteropathy between olmesartan and other ARBs were not significantly different, regardless of drug period after adjustment for age, sex, and comorbidities (all p > 0.05).

\section{Differences in clinical parameters and biochemical analysis}

A total of 3,758 patients underwent medical examinations 1 year before the initiation of ACEis or olmesartan and during drug use. Using propensity score matching, we matched 665patients in each drug group. Baseline data and changes in clinical and biochemical parameters use are shown in Table 4. Age, sex, income level, drug use period, follow-up duration, comorbidities, baseline anthropometric and biochemical parameters did not differ within the matched cohorts (all $p>0.05$ ). Baseline weight and BMI were $65.8 \mathrm{~kg}, 24.9 \mathrm{~kg} / \mathrm{m}^{2}$ in ACEis group, and $66.5 \mathrm{~kg}, 24.9 \mathrm{~kg} / \mathrm{m}^{2}$ in olmesartan group, respectively (all $p>0.05$ ). The decrease in body weight per year was greater in the ACEis than the olmesartan group $(-0.3 \mathrm{~kg} /$ year vs. $0.1 \mathrm{~kg} / \mathrm{year}$, respectively; $p<0.001)$. BMI also de-

Table 2. Risk over time: descriptive data

\begin{tabular}{|c|c|c|c|}
\hline Variable & ACEi & Olmesartan & ARB \\
\hline \multicolumn{4}{|l|}{ Person } \\
\hline$<1 \mathrm{yr}$ & 5,447 & 12,299 & 24,014 \\
\hline$\geq 1 \mathrm{yr}$ & 3,040 & 11,311 & 52,448 \\
\hline \multicolumn{4}{|c|}{ No. of events } \\
\hline$<1 \mathrm{yr}$ & 4 & 3 & 9 \\
\hline$\geq 1 \mathrm{yr}$ & 3 & 2 & 10 \\
\hline \multicolumn{4}{|c|}{ Incidence per 1,000 persons } \\
\hline$<1 \mathrm{yr}$ & 0.73 & 0.24 & 0.37 \\
\hline$\geq 1 \mathrm{yr}$ & 0.99 & 0.18 & 0.19 \\
\hline
\end{tabular}

ACEi, angiotensin converting enzyme inhibitor; ARB, angiotensin receptor blocker.

Table 3. Crude and adjusted odd ratios of intestinal malabsorption over time (ref: ACEi)

\begin{tabular}{|c|c|c|c|c|c|c|}
\hline \multirow{2}{*}{ Variable } & \multicolumn{3}{|c|}{ Crude odd ratio } & \multicolumn{3}{|c|}{ Adjusted odd ratio } \\
\hline & Value & $95 \% \mathrm{CI}$ & $p$ value & Value & $95 \% \mathrm{CI}$ & $p$ value \\
\hline \multicolumn{7}{|c|}{ Overall population } \\
\hline Olmesartan & 0.26 & $0.08-0.80$ & 0.020 & 0.33 & $0.10-1.09$ & 0.070 \\
\hline Other ARBs & 0.33 & $0.10-1.01$ & 0.070 & 0.34 & $0.14-0.83$ & 0.017 \\
\hline \multicolumn{7}{|c|}{ Treatment duration < 1 yr } \\
\hline Olmesartan & 0.33 & $0.07-1.48$ & 0.149 & 0.45 & $0.09-2.19$ & 0.326 \\
\hline Other ARBs & 0.51 & $0.16-1.66$ & 0.263 & 0.53 & $0.16-1.75$ & 0.295 \\
\hline \multicolumn{7}{|c|}{ Treatment duration $\geq 1 \mathrm{yr}$} \\
\hline Olmesartan & 0.18 & $0.03-1.07$ & 0.060 & 0.25 & $0.04-1.62$ & 0.147 \\
\hline Other ARBs & 0.19 & $0.05-0.70$ & 0.013 & 0.24 & $0.06-0.90$ & 0.034 \\
\hline
\end{tabular}

Adjusted with age, sex, income level, hypertension, dyslipidemia, heart failure, dementia, diabetes mellitus, autoimmune disease, chronic kidney disease, organ transplantation, and cancer.

ACEi, angiotensin converting enzyme inhibitor; CI, confidence interval; ARB, angiotensin receptor blocker. 
Table 4. Changes of clinical and biochemical parameters during drug use among matched cohort

\begin{tabular}{|c|c|c|c|}
\hline Variable & $\operatorname{ACEi}(n=665)$ & $\operatorname{Olmesartan}(\mathrm{n}=665)$ & $p$ value \\
\hline Age, yr & $61.5 \pm 11.5$ & $61.0 \pm 10.5$ & 0.658 \\
\hline Female sex & $210(31.6)$ & $211(31.7)$ & 1.000 \\
\hline Income level, deciles & $6.0 \pm 2.4$ & $5.9 \pm 2.5$ & 0.658 \\
\hline Initial examination time, yr & $2,006.7 \pm 2.1$ & $2,006.7 \pm 2.0$ & 0.979 \\
\hline Follow-up duration, yr & $2.4 \pm 0.8$ & $2.3 \pm 0.8$ & 0.614 \\
\hline \multicolumn{4}{|l|}{ Comorbidities } \\
\hline Hypertension & $561(84.4)$ & $573(86.2)$ & 0.395 \\
\hline Dyslipidemia & $269(40.5)$ & $274(41.2)$ & 0.823 \\
\hline Heart failure & $56(8.4)$ & $56(8.4)$ & 1.000 \\
\hline Dementia & $5(0.8)$ & $7(1.1)$ & 0.772 \\
\hline Diabetes mellitus & $224(33.7)$ & $205(30.8)$ & 0.291 \\
\hline Autoimmune disease & $11(1.7)$ & $13(2.0)$ & 0.837 \\
\hline Organ transplantation & $2(0.3)$ & 0 & 0.479 \\
\hline Ongoing cancer & $20(3.0)$ & $22(3 \cdot 3)$ & 0.875 \\
\hline Chronic kidney disease & $6(0.9)$ & $6(0.9)$ & 1.000 \\
\hline \multicolumn{4}{|l|}{ Systolic blood pressure } \\
\hline Baseline, $\mathrm{mmHg}$ & $134 \cdot 9 \pm 17 \cdot 9$ & $135 \cdot 0 \pm 16.5$ & 0.875 \\
\hline Change, $\mathrm{mmHg} / \mathrm{yr}$ & $-2.9 \pm 8.8$ & $-2.6 \pm 9.6$ & 0.605 \\
\hline \multicolumn{4}{|l|}{ Weight } \\
\hline Baseline, kg & $65.8 \pm 11.2$ & $66.5 \pm 11.2$ & 0.272 \\
\hline Change, kg/yr & $-0.3 \pm 1.6$ & $0.1 \pm 1.4$ & $<0.001$ \\
\hline Significant weight loss ${ }^{\mathrm{a}}$ & $21(3.2)$ & $13(2.0)$ & 0.224 \\
\hline \multicolumn{4}{|l|}{ Body mass index } \\
\hline Baseline, $\mathrm{kg} / \mathrm{m}^{2}$ & $24.9 \pm 3.0$ & $24.9 \pm 3.1$ & 0.870 \\
\hline Change, $\mathrm{kg} / \mathrm{m}^{2} / \mathrm{yr}$ & $-0.1 \pm 0.6$ & $0.0 \pm 0.6$ & $<0.001$ \\
\hline \multicolumn{4}{|l|}{ Total cholesterol } \\
\hline Baseline, mg/dL & $201.5 \pm 39.0$ & $201.4 \pm 38.7$ & 0.961 \\
\hline Change, mg/dL/yr & $-7.8 \pm 21.2$ & $-3.4 \pm 19.4$ & $<0.001$ \\
\hline \multicolumn{4}{|l|}{ Hemoglobin } \\
\hline Baseline, g/dL & $14.4 \pm 1.5$ & $14.4 \pm 1.5$ & 0.534 \\
\hline Change, g/dL/yr & $-0.2 \pm 0.6$ & $-0.2 \pm 0.6$ & 0.115 \\
\hline
\end{tabular}

Values are presented as mean $\pm \mathrm{SD}$ or number (\%).

ACEi, angiotensin converting enzyme inhibitor.

${ }^{\text {a }}$ Significant weight loss was defined as more than $5 \%$ loss of body weight compared with baseline

creased more in ACEi than olmesartan group (-0.1 kg/ $\mathrm{m}^{2} /$ year vs. $0.0 \mathrm{~kg} / \mathrm{m}^{2} /$ year, respectively; $\left.p<0.001\right)$. However, the proportion of patients with significant weight loss did not differ between the ACEis and olmesartan groups (3.2\% vs. $2.0 \%$, respectively; $p=0.224$ ). The decrease of total cholesterol is higher in ACEi than olmesartan group (-7.8 mg/dL/year vs. $-3.4 \mathrm{mg} / \mathrm{dL} /$ year, re- spectively; $p<0.001)$.

\section{DISCUSSION}

Our nationwide cohort study found that olmesartan use was not associated with a higher incidence of intestinal 
malabsorption than ACEis or other ARBs in Koreans. The subgroup comparisons of clinical and biochemical parameters before initiation and during drug use revealed that decrease of BMI or weight loss is more apparent in ACEis group than in olmesartan group. The risk of decrease in cholesterol levels, which is a feature of celiac disease, was not evident in patients using olmesartan, either.

Following the study of Rubio-Tapia et al. [2], which suggested an association between olmesartan and spruelike enteropathy of unknown causes, several similar cases have been reported. Ianiro et al. [14] identified several shared symptoms and disease courses in patients suspected to have olmesartan-associated enteropathy. Most cases were old Caucasians who had been exposed to olmesartan for more than 6 months. A gluten-free diet did not improve the symptoms in the most patients; however, the symptoms resolved following the cessation of olmesartan. Moreover, several cases of profound sprue-like enteropathy associated with other ARBs have been reported $[5,6]$.

A national survey conducted in France studied 36 patients suspected to have olmesartan-associated enteropathy [15]. Although the study did not include a deliberate rechallenge test, interruption of the drug was followed by remission, and reintroduction of the drug resulted in relapse. In the study using the French National Health Insurance Claim database, Basson et al. [8] found that olmesartan was associated with an increased risk of hospitalization for intestinal malabsorption and celiac disease. The risk of enteropathy was higher in patients with a longer duration of treatment. However, according to the Naranjo probability scale, a cause-effect relationship is only probable because none of patients with suspected olmesartan-associated enteropathy was exposed to a rechallenge test [16]. Following a search of the Mayo Clinic endoscopy database, Greywoode et al. [17] concluded that olmesartan-associated enteropathy is a rare adverse drug effect, and that milder presentations causing diarrhea in a substantial number of outpatients are unlikely. The large randomized clinical trial found no significant differences in the rate of gastroenterological adverse events and diarrhea between olmesartan and placebo groups with DM [18]. The cohort study using a United States integrated insurance and laboratory claim database found no difference in olmesartan- and other ARB-associated hospital admissions in patients with DM, either [19]. The mechanisms underlying the putative association between olmesartan and spruelike enteropathy are not known. Previous case reports have shown that symptoms appear months to years after olmesartan initiation. Intestinal biopsies have revealed that villous atrophy and mucosal inflammation improve after drug discontinuation, but are not affected by a gluten-free diet. Moreover, IgA transglutaminase antibodies are notably absent. A cell-mediated or delayed hypersensitivity reaction, potentially associated with the human leukocyte antigen-DQ cell surface receptor type 2, has been proposed [2]. The HLA-DQ2 or HLA-DQ8 haplotype is present in $70 \%$ of reported patients [3]. The fact that the prevalence of $\mathrm{DQ}_{2} / \mathrm{DQ} 8$ is estimated to be $30 \%$ to $40 \%$ in normal Western populations [20] and that reported cases have a higher prevalence of a susceptible genetic background for celiac disease, suggests a possible role for genetics in olmesartan-induced enteropathy.

The actual prevalence of celiac disease in Northeast Asia, including Korea and Japan, is not known. To date, celiac disease has only been described in two case reports in Korea [21,22]. One reason for the low prevalence of celiac disease is the low prevalence of HLA DQ2/DQ8 haplotypes in these countries. In contrast to the relatively high prevalence of the haplotypes in Western countries and India (more than 20\%), the prevalence of the haplotypes is less than $5 \%$ in Japan and Korea [9]. Variance in the HLA DQ2 and DQ8 haplotypes among countries may explain geographic differences in olmesartan-associated enteropathy. However, possible adverse effects induced by olmesartan should not be overlooked. Although our findings suggest that there is no association between olmesartan and the incidence of enteropathy in the Korean population, awareness of olmesartan-associated enteropathy may help physicians to avoid misclassification of the disorder, and once identified, the intervention (switching antihypertensive medication) is simple and effective.

The reason why enteropathy was more prevalent in patients using ACEi than patients using ARBs is not clear. This result should be interpreted with caution due to a relatively small number of patients developing intestinal malabsorption. Patients using ACEi had more $\mathrm{DM}$, heart failure, autoimmune disease, history of or- 
gan transplantation and ongoing cancer compared with those using olmesartan. Although we adjusted all these comorbidities and income levels, our findings may result from differences in baseline characteristics which were not adjusted. In order to more accurately correct baseline characteristics, we performed additional analysis with propensity score matching of various results from health examination. In this analysis, we also found that body weight was more decreased in ACEi group. However, there was no difference in significant body weight loss, which might affect the prognosis in elderlies [12]. Further researcher is needed on antihypertensive medication and subclinical weight changes in Asians.

The strength of this study is the first to identify the relationship between olmesartan and intestinal malabsorption in Asians by analyzing nationally representative cohort with relatively large sample size and long follow-up duration. The limitations of our study include the observational nature of the study design and our inability to access complete medical records. Because we used NHIS claim data, it is likely that missing diagnoses or misclassification of intestinal malabsorption or other comorbidities occurred as a result of diagnostic or coding errors, which could cause misinterpretation of the adverse drug effects. Celiac disease is rare in Korea; thus, it may have been underdiagnosed in our sample. Furthermore, milder presentations of drug-associated enteropathy may have been overlooked by physicians and patients. We tried to correct these errors by comparing body weight, hemoglobin, and cholesterol in the drug groups, which can be affected by hidden intestinal malabsorption [23]. The NHIS-NSC 2002 to 2013 is a random sample of $2 \%$ of the general Korean population. Although this national cohort is a relatively small proportion of the population, it represents a stratified random sample of the Korean population with a long-term follow up. Further prospective larger-scale studies are required to investigate olmesartan-associated enteropathy and other adverse effects in Asians.

In conclusions, we found no evidence to suggest that olmesartan is associated with intestinal malabsorption or significant body weight loss in the general Korean population.

\section{KEY MESSAGE}

1. Olmesartan use does not increase the risk of intestinal malabsorption in the general Korean population compared to angiotensin converting enzyme (ACE) inhibitor use.

2. Olmesartan use was not associated with the significant body weight loss in the general Koean population compared to ACE inhibitor use.

\section{Conflict of interest}

No potential conflict of interest relevant to this article was reported.

\section{Acknowledgments}

This research was supported by a grant of the Korea Health Technology R\&D Project through the Korea Health Industry Development Institute (KHIDI), funded by the Ministry of Health and Welfare, Republic of Korea (grant number: HI16Co992) and supported by a grant of the Korea Health Technology R\&D Project through the Korea Health Industry Development Institute (KHIDI), funded by the Ministry of Health and Welfare, Republic of Korea (grant number: $\mathrm{HI}_{4} 4 \mathrm{C}_{3201}$ ).

\section{REFERENCES}

1. Scott LJ, McCormack PL. Olmesartan medoxomil: a review of its use in the management of hypertension. Drugs 2008;68:1239-1272.

2. Rubio-Tapia A, Herman ML, Ludvigsson JF, et al. Severe spruelike enteropathy associated with olmesartan. Mayo Clin Proc 2012;87:732-738.

3. Burbure N, Lebwohl B, Arguelles-Grande C, Green PH, Bhagat G, Lagana S. Olmesartan-associated sprue-like enteropathy: a systematic review with emphasis on histopathology. Hum Pathol 2016;50:127-134.

4. Bhat N, Anupama NK, Yelsangikar A, Vizhi K. Olmesartan-related sprue-like enteropathy. Indian J Gastroenterol 2014;33:564-567.

5. Cyrany J, Vasatko T, Machac J, Nova M, Szanyi J, Kopacova M. Letter: telmisartan-associated enteropathy. Is there any class effect? Aliment Pharmacol Ther 2014;40:569-570.

6. Herman ML, Rubio-Tapia A, Wu TT, Murray JA. A case of 
severe sprue-like enteropathy associated with valsartan. ACG Case Rep J 2015;2:92-94.

7. US Food and Drug Administration. Safety information July 2013 [Internet]. Silver Spring (MD): US Food and Drug Administration, c2016 [cited 2017 Sep 11]. Available from: https://www.fda.gov/downloads/Drugs/DrugSafety/ UCM359496.pdf.

8. Basson M, Mezzarobba M, Weill A, et al. Severe intestinal malabsorption associated with olmesartan: a French nationwide observational cohort study. Gut 2016;65:16641669 .

9. Cummins AG, Roberts-Thomson IC. Prevalence of celiac disease in the Asia-Pacific region. J Gastroenterol Hepatol 2009;24:1347-1351.

10. Kwon S. Payment system reform for health care providers in Korea. Health Policy Plan 2003;18:84-92.

11. Lee J, Lee JS, Park SH, Shin SA, Kim K. Cohort profile: the National Health Insurance Service-National Sample Cohort (NHIS-NSC), South Korea. Int J Epidemiol 2017;46:e15.

12. Wallace JI, Schwartz RS, LaCroix AZ, Uhlmann RF, Pearlman RA. Involuntary weight loss in older outpatients: incidence and clinical significance. J Am Geriatr Soc 1995;43:329-337.

13. Stuart EA. Matching methods for causal inference: a review and a look forward. Stat Sci 2010;25:1-21.

14. Ianiro G, Bibbo S, Montalto M, Ricci R, Gasbarrini A, Cammarota G. Systematic review: sprue-like enteropathy associated with olmesartan. Aliment Pharmacol Ther 2014;40:16-23.
15. Marthey L, Cadiot G, Seksik P, et al. Olmesartan-associated enteropathy: results of a national survey. Aliment Pharmacol Ther 2014;40:1103-1109.

16. Naranjo CA, Busto U, Sellers EM, et al. A method for estimating the probability of adverse drug reactions. Clin Pharmacol Ther 1981;30:239-245.

17. Greywoode R, Braunstein ED, Arguelles-Grande C, et al., Olmesartan, other antihypertensives, and chronic diarrhea among patients undergoing endoscopic procedures: a case-control study. Mayo Clin Proc 2014;89:1239-1243.

18. Haller H, Ito S, Izzo JL Jr, et al. Olmesartan for the delay or prevention of microalbuminuria in type 2 diabetes. $\mathrm{N}$ Engl J Med 2011;364:907-917.

19. Padwal R, Lin M, Etminan M, Eurich DT. Comparative effectiveness of olmesartan and other angiotensin receptor blockers in diabetes mellitus: retrospective cohort study. Hypertension 2014;63:977-983.

20. DiGiacomo D, Santonicola A, Zingone F, et al. Human leukocyte antigen DQ2/8 prevalence in non-celiac patients with gastrointestinal diseases. World J Gastroenterol 2013;19:2507-2513.

21. Gweon TG, Lim CH, Byeon SW, et al. A case of celiac disease. Korean J Gastroenterol 2013;61:338-342.

22. Hwang IK, Kim SH, Lee U, et al. Celiac disease in a predisposed subject (HLA-DQ2.5) with coexisting Graves' disease. Endocrinol Metab (Seoul) 2015;30:105-109.

23. Malandrino N, Capristo E, Farnetti S, et al. Metabolic and nutritional features in adult celiac patients. Dig Dis 2008;26:128-133. 